\title{
An Android based Automatic Irrigation System using a WSN and GPRS Module
}

\author{
A. Mahesh Reddy ${ }^{1 *}$ and K. Raghava Rao ${ }^{2}$ \\ 'Embedded Systems, Department of ECM, K. L. University, Andhra Pradesh, India; alumuru.mahesh@gmail.com \\ ${ }^{2}$ Department of ECM, K. L. University, Andhra Pradesh, India; raghavarao@kluniversity.in
}

\begin{abstract}
Background/Objectives: This paper presents an automatic irrigation system to provide water to the farms based on soil and temperature conditions using an android application, WSN and GPRS modules. Methods/statistical Analysis: An algorithm is developed such that soil moisture sensor and temperature sensor values are continuously fed to Arduino UNO micro controller. The sensor information is compared with the threshold values and based on that, decision will be taken to water the crops. The system is equipped with the photovoltaic panels and dual communication is established based on cellular-internet interface for continuous inquiry of data by the user. We have also developed an android mobile application for intercepting the data generated. For that purpose, we can also employ a web site. Findings: Because of system's energy sovereignty, low cost and relatively more amounts of underground water saving, this system is preferable at water scarcity locations like desert areas. Conclusion: This irrigation system has been working with high efficiency and top speed. This system sends message to the user whenever sensors exceed there threshold value, by this system every user can understand the soil conditions and controls the system too manually, if needed.
\end{abstract}

Keywords: Automatic Irrigation, Arduino UNO, GSM Module, Soil Moisture Sensor, Temperature Sensor

\section{Introduction}

Agriculture plays an imperative role in the economy of India. Moreover $58 \%$ of the Indian rural population depends on agriculture as it is the means of subsistence and main asset for secured livelihood. Agriculture is the biggest contribution to the Indian Gross Domestic Product (GDP). In 2009, a study has been released by the NASA, showing the level of ground water

across the northern India, that more than one foot of water level has been reducing per decade and the main cause behind it was human activity. One of the main reasons for recession of ground water is agriculture, where the farms are fetching more water from the ground level as the rainfall through the country changes rapidly. Over all $92 \%$ of ground level water is used alone for agriculture and $49 \%$ of that water is from the underground water resources. This consumption of water will dominantly continue because of growth in population and also the increase in the demand of food sources. So there is an essential need to constitute the scenario based on science and technology for feasible management of water.

As the science and technology increases rapidly there are numerous methods in irrigating the water for various crops. For instance, water status of the plant and irrigation were monitored by the canopy temperature. Canopy temperature was seized with the help of thermal imaging $^{1}$. Another method was Crop Water Stress Index (CWSI). Observational CWSI ${ }^{2}$ was defined 30 years ago and by using these methods the schedule crop irrigation has been done. The ratio of deficit values was calculated later by using infrared canopy temperature measurement, ambient air temperature and atmospheric vapour pressure. This value helps to regulate drip irrigation ${ }^{3}$ for crops whenever needed. More authors described usage of remote canopy temperature using infrared thermometer to automatically water the cotton crop irrigation.

Volumetric water content of soil information is gathered by the dielectric moisture sensors and by using this information the irrigation system can be automated as it

${ }^{*}$ Author for correspondence 
may control the actuators and ground level water instead of pre-arranged irrigation plan at specific duration of time in a particular day. Controller based irrigation is also developed to get a value of solenoid and apply watering to the plants when the level of volumetric water decreases below a set point ${ }^{4}$.

Monitoring the moisture level of the soil helps in determining when to irrigate and it significantly helps to improve the water level conversion and conservation of energy, maximization of yield, improvement of water quality and also in reducing the erosion of the soil. In irrigation, a specific crop has a different factor which determines the requirements of water. Irrigation of the plant typically requires actual weather data including relative humidity, speed of the wind and crop factors such as growth stage, variety of the plant, density of the plants in the field, properties of the soil and disease control. Watering is done by the estimation of the plant based on Evapo Transpiration (ET) ${ }^{5}$. System based on ET has been developed and $42 \%$ of the water has been saved on time based irrigation. ET-based irrigation is more sustainable.

The other method of diminishing of water for irrigation is developed with a Multi-Source Information Fusion technology (MSIF) based on entropy ${ }^{6}$. Irrigation was done with sprinklers through GPS; Bluetooth technology is used for communication. Many others are using different controllers to trace the sensors data and implementing through Zigbee ${ }^{7}$. Another effective method of watering is micro irrigation or drip irrigation. It employs drip emitter which slowly allows water directly to the root zone of the crop. A pressurized tubing system present in the drip irrigation system runs along crop rows and these are fitted by nozzles at a particular distance as water drip from these emitters. Micro irrigation is another method which is mainly used in vine and tree crops. It is also popular where the water level is limited or recycled water is used for irrigation and it is named as row crop irrigation. The specific feature of micro-irrigation is high irrigation frequency and the water is specifically applied to the crops root zone.

An electromagnetic sensor is used to know the moisture of soil and it helps in achieving an irrigation system where $53 \%$ of water is saved when compared with the sprinkles irrigation system in a large area ${ }^{8}$. A system is also developed for the cultivation of barley in large areas of land through decision support software which is integrated to the wireless sensor networks aiding in sprinkler nozzles controllability. Each sensing station is equipped with a data logger with two soil water reflectometers, Bluetooth for communication and a soil temperature sensor. By using this network information and the position of the irrigation system by differential GPS, the sprinklers are controlled by software?

By using Zigbee technology, automated remote monitoring system is developed which is used for wireless monitoring in the field and user may see accurate variations ${ }^{10}$. To improve the cultivation production precision agriculture a broad system is designed, which also diminishes the usage of watering for agriculture ${ }^{11}$.

In this paper we propose the improvement of distribution of water in an automated irrigation system based on an Arduino platform and wireless communications. The main aim is to reduce the usage of underground water with automatic irrigation which is implemented through photovoltaic panels. Initially user will set the sophisticated soil moisture sensor and temperature sensor under the roots of plant. The information received by these sensors is transmitted to the microcontroller. The micro controller compares the received values with the threshold values. If the sensor values reaches the threshold values, then user will receive an SMS alert to mobile. Then user can switch ON the motor through an android application or through web. The internet connection by the user allows continuous data inspection in real time android mobile application which we have developed, and values of the sensors may be observed graphically and can be automatically stored in the web server. The prototype of the system architecture is developed by integrating the required modules.

\section{System Design}

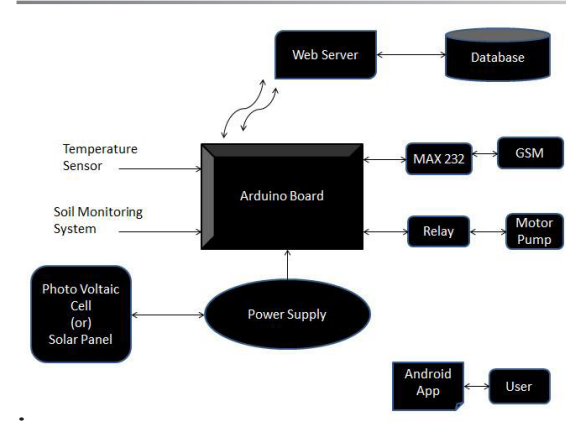

Figure 1. System Architecture.

Figure 1 depicts system architecture, an android based automatic irrigation system. The architecture consists of following modules. 


\subsection{LM35}

To sense the parameters of temperature in irrigation we use LM35. The LM35 is a series \& precision integratedcircuit temperature sensor, which can be used with single power supply and also Celsius (Centigrade) temperature, is directly proportional to the output voltage of the device. The main advantage of this over linear temperature sensor is that, the user need not diminish the large constant voltage from the output to obtain the convenient centigrade scaling. Inexpensiveness is guaranteed by trimming and calibration is done at the water level. LM35 devices make easy interfacing and also control the circuit due to specific characters like, low-output impedance, continuous output etc.

\subsection{Soil Moisture Sensor}

Calculating the soil moisture in the fields is important to help the users improve irrigation system efficiently. Soil moisture sensor is also a capacitive type and by knowing its exact information, farmers may use less water for irrigating a crop. The quality of the crop and production can be increased by management of soil moisture during critical stages of plant growth. Volumetric water content is calculated indirectly by soil moisture sensor using different parameters of the soil like dielectric constant, electrical resistance, neutrons interaction. Relational values between the measured property and calibrated soil moisture may vary depending on different environmental factors like type of soil, temperatures, or electric conductivity.

\subsection{Solar Panel}

Solar panel is an electrical device which can convert the light energy precisely into electricity by photovoltaic effect. These semiconductors such as silicon are made of special materials of the cell. Electrical characteristics of a photo electric cell namely, current, voltage or resistance fluctuate when directly exposed to light. Solar panel is made of blocks, consistently photovoltaic modules.

\subsection{Arduino UNO}

Arduino is an open source device for hardware and software integration. These devices help to sense the objects and control them in the real world. Arduino gives set of digital and analogy input/output pins which can be interfaced to distinctive developed boards ("shields") and different circuits. Serial communication interfaces, including USB are required for loading programs from the system. Arduino board gives an integrated development environment (IDE) for programming the microcontroller in processing the project, which incorporates support of different languages. Basic Arduino UNO is shown in Figure 2.

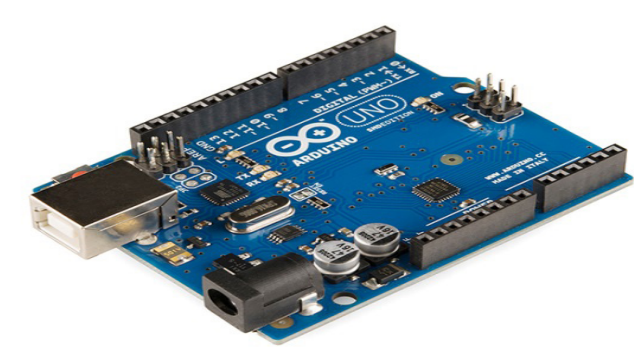

Figure 2. Arduino UNO.

\subsection{Power Supply}

Power supply device's primary function is to supply AC/ DC electrical energy to electrical load. The Arduino is connected to the RPS. The AC input i.e., 230v from main source is connected to the circuit \& the voltage stepped down by the transformer to $12 \mathrm{v}$ is supplied and is used as a rectifier. Output which is obtained from the rectifier is a fluctuating DC voltage which is supplied to a filter to remove any unwanted $\mathrm{AC}$ components. In order to get constant DC voltage, voltage regulator has been used.

\subsection{Relay}

Relays are simple switches which can be operated both mechanically and electrically in which electromagnet and also set of contacts are present. Relay works when there is a low-power signal and this relay information is used to control the circuits. It is helpful in a scenario, where a number of circuits are controlled by a single signal. They are used as switches in which the incoming signals are switched from one source to different destinations. Relays are also used to perform Boolean and other logical operations in a computer. High end application of relay is driven by the high power and such relay is called a contactor.

\subsection{MAX232}

The MAX 232 is an integrated circuit which converts the signal TIA-232(RS-232) serial port to the signal which is 
suitable to use it in TTL digital compatible logical circuits. The MAX232 is a combination of transmitter/receiver and it commonly converts over TX, RX, RTS and CTS signals.

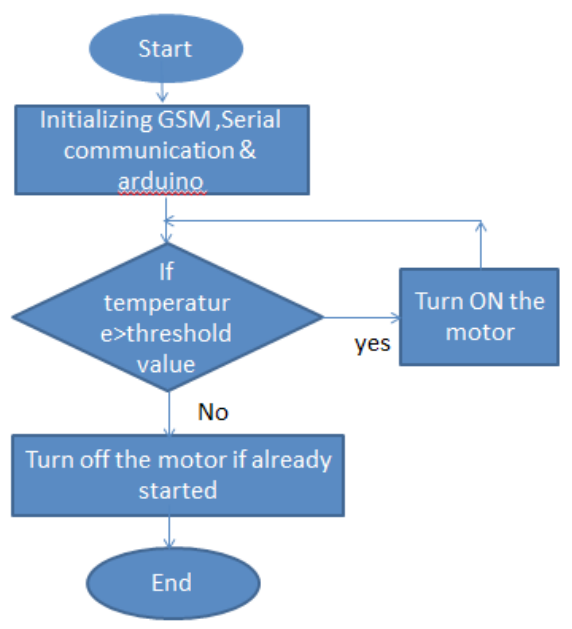

Figure 3. Flow chart for controlling motor through GSM module.

\subsection{GSM/GPRS SIM900a Module}

GSM/GPRS is also known as resilient Quad-band mobile call up, which mainly performs on a different frequency of $850 / 900 / 1800 / 1900 \mathrm{MHZ}$ and also utilized to get access through the internet as well as oral communication as it contains an amplifier and a little loud speaker as combination and it also helpful in SMS's ${ }^{12}$.

\subsection{Android Application}

An Android application is developed, which helps mainly in operating and controlling the devices by the user ${ }^{13}$. The values from the sensors are received and displayed continuously in an android application. By using this information user can manually operate the system by switching ON/OFF based on the threshold values.

\subsection{Web Application}

For real-time monitoring of irrigation user can operate through graphical user interface GUI to control the system. This web application displays the sensor values and a switch for ON/OFF button activity. The data collected is deposited in the database for future reference. The data is refreshed continuously to update the information. We can utilize web application through the PC/android mobile.

\section{Flow Chart for Controlling a Motor through GSM Module}

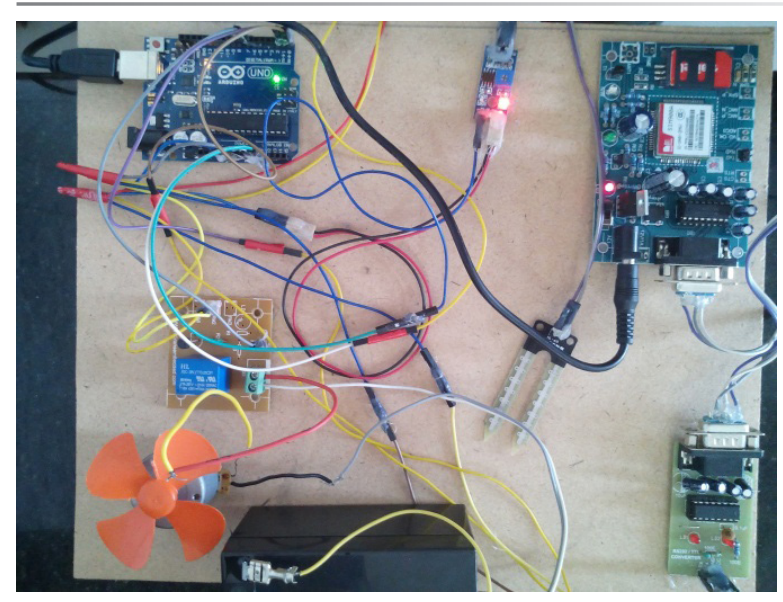

Figure 4. Snap shot of proposed system hardware.

We can control the motor through GSM module, if temperature sensor reading is higher than the threshold value then turn $\mathrm{ON}$ the motor, otherwise turn OFF the motor if already started or else do nothing. This flowchart is shown in Figure 3.

\section{Working of Automatic Irrigation System}

The soil moisture sensor and temperature sensor are connected to analogy pins of an Arduino board. 5v power supply is generated from USB cable which is connected to pc or by the adaptor of an Arduino board. A solar panel is used to charge the battery continuously during day time to provide power to the $12 \mathrm{v}$ battery, it will be charged continuously until sunset. After sunset the charged power should be discharged continuously, when user can operate the motor. By using this solar panel we can optimize usage of power. First of all GSM/GPRS module is initialized and waits until it connects to the network. $12 \mathrm{v}$ power supply is scheduled to the GSM/GPRS module through adaptor. The red light indicator illustrates GSM/GPRS is ON and white light indicator illustrates strength of the network. When the white light indicator blinks slowly it coveys that GSM/GPRS has strong signal strength.

The data sensed by soil moisture sensor and temperature sensor is transmitted to micro-controller. Thresholds values are programmed into an Arduino board initially. So, the values which are received from sensors are com- 
pared with threshold values time to time. Here two conditions should be satisfied viz., if the readings from the temperature sensor are more than the predetermined value and the soil moisture reading is lesser than the predetermined value. Then a SMS alert is sent to the user by GSM/GPRS module. Then the motor pump is switched ON by the user with the aid of an android application or through real time website. All the data gathered is reserved in the web server for future references ${ }^{14}$.

\section{Proposed Method Hard Ware and Results}

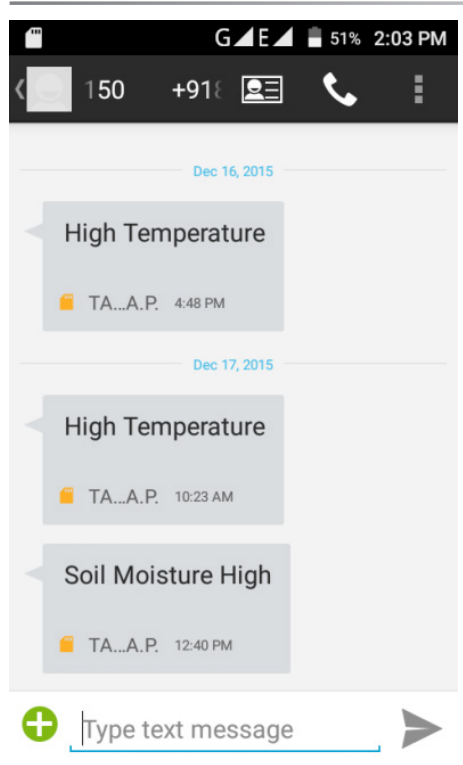

Figure 5. SMS alert to user.

The complete hardware unit of an Automatic irrigation system can be developed by integrating GSM/GPRS module, temperature and soil moisture sensors, relay, battery, RS232 and solar panel. The user want to control the motor pump through an android application and a web application, when he receives an SMS (Short Message Service) alert via GSM/GPRS module. Figure 4 shows the temperature and soil moisture sensors will send the sensors information to Arduino continuously. For those input values which crosses the threshold, user will receive an SMS alert via GSM/GPRS module. Then user will ON the motor through an android application or web application and vice versa.

Figure 5 explains about the situation of, when the threshold values and recorded sensor values are compared with each other and the resultant value, whether HIGH/LOW is notified to the user.

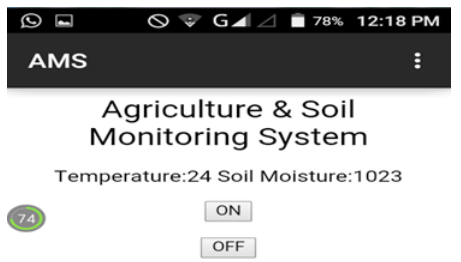

Figure 6. An android application for user.

Figure 6 explains the android application in the user mobile devices through which he can operate the motor by switching ON/OFF button.

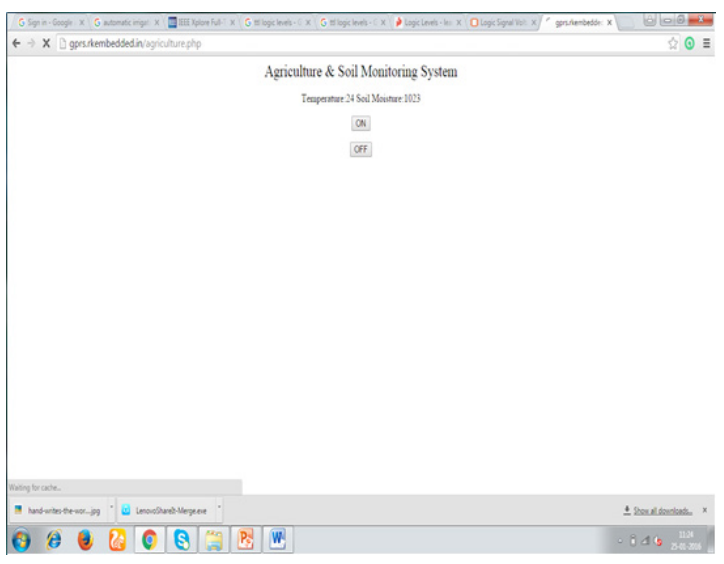

Figure 7. A real time web page application to user.

Figure 7 explains the real time web page where the user can operate the motor through the mobile or personal computer (PC).

\section{Conclusion}

An android automated irrigation system is cost effective and feasible. A farmer should visit his agricultural land frequently to test moisture content and temperature and to avoid this, android automatic system is developed; it displays the values of the sensors continuously in an android application and on a web page and user can operate them from any place. When user receives an SMS alert through GSM/GPRS module then user switches ON the motor. It is really an efficient and an economical way to diminish wastage of water and human power in irrigation 
field. This system is efficient, reliable and helps in saving of water and electricity, when compared to the other systems.

\section{Acknowledgements}

We are grateful for the constant help and assistance provided by K L University. We acknowledge the ESSN (Embedded System and Sensor Networks) research group of our University

\section{References}

To refer a research article:

1. Wang X, Yang W, Wheaton A, Cooley N, Moran B. Efficient registration of optical and IR images for automatic plant water stress assessment. Comput Electron Agr. 2010 Nov; 74(2):230-7.

2. Yuan G, Luo Y, Sun X, Tang D. Evaluation of a crop water stress index for detecting water stress in winter wheat in the North China Plain. Agr Water Manag. 2004 Jan; 64:29-40.

3. Erdem Y, Arin L, Erdem T, Polat S, Deveci M, Okursoy H, Gultas HT. Crop water stress index for assessing irrigation scheduling of drip irrigated broccoli (Brassica oleracea L. var. Italic). Agr Water Manag. 2010 Dec; 98(1):148-56.

4. Nemali KS, Van Iersel MW. An automated system for controlling drought stress and irrigation in potted plant. Scientia Horticulture. 2006 Nov; 110(3):292-7.

5. Davis SL, Dukes MD. Irrigation scheduling performance by evapotranspiration-based controllers. Agricult Water Management. 2010 Dec; 98(1):19-28.

6. Zhao L-M, Liu H-P, Bing Z. The system of water-saving irrigation based on WSN and MSIF. International Journal of Computer Science Issues (IJCSI). 2012 Nov; 9(6):47480.

7. Zhou Y, Yang X, Wang L, Ying Y. Low cast design of wireless irrigation system based on ZigBee technology Networks Security. 2009 NSWCTC '09 International Conference on Wireless Communications and Trusted Computing.2009 Apr. p. 572-5.

8. Blonquist JM, Jones SB, Robinson DA. Precise irrigation scheduling for turfgrass using a subsurface electromagnetic soil moisture sensor . Agricult Water Management. 2006 Jul; 84:153-65.

9. Kim Y, Evans RG, Iversen WM. Remote sensing and control of an irrigation system using a distributed wireless sensor network. IEEE Trans Instrum Meaurement. 2008 Jul; 57(7):1379-87.

10. Chavan $\mathrm{CH}$, Karande PV. Wireless monitoring of soil moisture, temperature and humidity using zig bee in agriculture. International Journal of Engineering Trends and Technology (IJETT). 2014 May; 11(10):1-5.

11. Vijaya kumar S, VishnuVardhan D. Wireless Network Sensors for Precise Agriculture Monitoring. (IJIRSE) International Journal of Innovative Research in Science and Engineering. 2011 Mar; 307-10.

12. Dalip, VK. Effect of Environmental Parameters on GSM and GPS. Indian Journal of Science and Technology. 2014 Aug; 7(8):1183-8.

13. Prakash M, Gowshika U, Ravichandran T. A Smart Device Integrated with an Android for Alerting a Person's Health Condition: Internet of Things. Indian Journal of Science and Technology. 2016 Feb; 9(6):1-6 .

14. Nallani S, Berlin Hency V. Low Power Cost Effective Automatic Irrigation System. Indian Journal of Science and Technology. 2015 Sep; 8(23):1-6. 